\title{
STUDIES ON INFECTIOUS MONONUCLEOSIS. II. THE RELATIONSHIP OF THE ORGANISMS OF THE GENUS LISTERELLA TO THE DISEASE, AS STUDIED BY THE AGGLUTINATION REACTION
}

\author{
By CHARLES A. JANEWAY and GUSTAVE J. DAMMIN \\ (From the Medical Clinic of the Peter Bent Brigham Hospital and the Departments of Medicine \\ and Bacteriology and Immunology, Harvard Medical School, Boston)
}

(Received for publication November 28, 1940)

Since the discovery that organisms of the genus Listerella (Bact. monocytogenes) would produce a mononucleosis in rabbits, there has been speculation as to their possible etiological relationship to infectious mononucleosis in man $(1,2)$. Nyfeldt (3) cultivated these organisms from the blood in 3 out of 33 cases of what seems to have been infectious mononucleosis. However, these cases were not proven by the sheep cell agglutination test, and proof of the etiologic rôle of the organisms cultivated was lacking since Nyfeldt failed to test his patients for the development of agglutinins. Schmidt and Nyfeldt (4) have recently reported the successful cultivation of Listerella from the cerebrospinal fluid in 5 cases of infectious mononucleosis, all of whom gave positive agglutination reactions for sheep cells in the blood. Pons and Julianelle (5) have also reported the isolation of a member of the genus Listerella from the blood of a case of the disease, but the patient failed to show agglutinins for the organism 6 weeks later. Cultures of lymph nodes removed for study have yielded equivocal results. Baldridge and his co-workers (6) removed nodes from 6 patients, obtaining diverse results, but did isolate small "diphtheroids" from 2, which actually might have been Bact. monocytogenes. Wising (7), whose studies led him to believe that infectious mononucleosis was a virus disease, removed nodes from 3 patients and in none of these cases did he demonstrate organisms on smear or culture.

No serologic studies dealing with this question have been reported, except for those of Kolmer (8). He showed that organisms of the Listerella group lacked any antigen capable of eliciting the formation of syphilis reagins or of sheep cell agglutinins in rabbits. Since the agglutination of sheep cells is characteristic of the sera of most patients with infectious mononucleosis, and false positive Wassermann reactions are obtained in a few cases, he brought this forward as evidence against the etiologic rôle of Bact. monocytogenes in this disease.

These conflicting reports have led us to undertake a study of the development of antibodies against organisms of the Listerella group in the sera of patients with infectious mononucleosis. Rabbits infected with these organisms by intravenous injection develop high titers of agglutinins very rapidly (9), so that, in this animal at least, the Listerella are highly effective antigens. Thus, it seems likely that human beings would develop high titers if they became infected. Such was the case in a patient with Listerella meningitis, reported by Carey (10). The titer rose to $1: 640$, but fell to zero 2 weeks after recovery. However, Carey noted the strong agglutination of his organism by normal horse serum and, since his patient was treated with antimeningococcus horse serum, the validity of the positive agglutination test is open to question, particularly since it disappeared so rapidly. Furthermore, Julianelle and Pons (11) have shown that local infection in rabbits (conjunctivitis) protects against reinfection, although antibodies are rarely found in the blood. Therefore, it is conceivable that if infectious mononucleosis represents a localized form of $L$ isterella infection, agglutinins might never appear.

Seastone (2) and Julianelle (11) have both demonstrated the existence of two distinct serological groups among the organisms of this genus. Our own work with seven strains confirms this finding, although we have shown that there is considerable antigenic overlapping (9). Among our seven strains we were fortunate enough to have two strains, one representative of each group, which had been obtained from human cases of 
meningitis. These were chosen for use as antigens in a study of the agglutinins for Listerella in the sera of patients with infectious mononucleosis.

\section{METHODS}

Cases. Control sera were obtained from patients on the wards of the hospital and from several of our associates. The conditions from which these patients were suffering are tabulated with the results. Cases of infectious mononucleosis were observed both on our own wards and at the Stillman Infirmary of the Department of Hygiene, Harvard University. 1 Cases were diagnosed on the basis of clinical findings, the presence of abnormal lymphocytes and a preponderance of mononuclear cells in the blood smear, and the development of a positive sheep cell agglutination test during the course of the disease. Sera were obtained over as long a period as possible so as to follow cases during the recovery period as well as during the acute phase of the disease.

\section{Agglutination tests}

Reagents. Fresh 20 -hour 0.2 per cent dextrose beef heart infusion broth cultures were used. These were adjusted to $\mathrm{pH} 7.4$ to 7.6 with $\mathrm{N} / 1 \mathrm{NaOH}$ just before use. Two strains were used: $C . H$. obtained by Carey in $1936,{ }^{2}$ from a child with meningitis, and $U M$. obtained by Dr. Theodore Steinberg in 1940 at the Chelsea Memorial Hospital 2 from a man with meningitis. C. $H$. be-

1 We are indebted to the members of the staff of this department, particularly to Drs. M. Murray, A. Kranes, and $E$. Eppinger, for their cooperation in this work.

2 The authors wish to thank Drs. C. V. Seastone and Carl Ten Broeck for sending strain $C$. $H$. from the collection at the Rockefeller Institute for Medical Research, Department of Animal and Plant Pathology, Princeton, New Jersey, and Mrs. Cornelia Dingle, Bacteriologist, longs to Julianelle's ruminant group, $U M$. to his rodent group serologically. All sera were taken with sterile precautions and stored at $5^{\circ} \mathrm{C}$. until used.

Tests. To $0.5 \mathrm{cc}$. of each serum dilution was added 0.5 cc. of fresh neutralized culture in a small test tube. The mixture was thoroughly shaken, the tubes placed in a $37^{\circ} \mathrm{C}$. water bath for 2 hours, and then in the refrigerator overnight. Readings were made the following morning. The sera of both controls and patients were tested against the same batch of antigen on the same day so as to maintain complete uniformity of procedure.

Sheep cell agglutination tests. The standard technique of Paul and Bunnell (12) was used. Sera were inactivated for 20 minutes at $56^{\circ} \mathrm{C}$. To each tube was added $0.5 \mathrm{cc}$. of serum dilution, $0.5 \mathrm{cc}$. of 2 per cent fresh washed sheep cells, and $1.0 \mathrm{cc}$. of sterile physiological saline. The tubes were shaken thoroughly, placed in the water bath at $37^{\circ} \mathrm{C}$. for 1 hour, and then in the refrigerator overnight. The next morning each tube was inverted three times and the agglutination read as ++++ if all cells were agglutinated, as + if just a few clumps could be seen with the naked eye. Wassermann and Hinton tests were performed according to the standard technique by. Miss Ruth Klabe, hospital serologist.

\section{RESULTS}

The results have been recorded in tabular form for convenience. In all the tables both the serum dilution used and the final dilution of serum after mixture with the antigen are recorded for comparison with the results of other workers, some of whom use one figure, some the other.

Table I shows the results of the sheep cell agglutination test on the control sera. It will be Joseph Pratt Diagnostic Hospital, Boston, for making strain $U M$. available to us.

TABLE I

Control sera. Sheep cell agglutination tests

\begin{tabular}{|c|c|c|c|c|c|c|c|c|c|c|}
\hline \multirow{3}{*}{ Serum } & Serum dilution $\ldots \ldots \ldots \ldots \ldots \ldots$ & $1: 2$ & $1: 4$ & $1: 8$ & $1: 16$ & $1: 32$ & $1: 64$ & $1: 128$ & $1: 256$ & \multirow{3}{*}{$\begin{array}{c}\text { Titer } \\
\text { (Final dilution) }\end{array}$} \\
\hline & Final dilution $\ldots \ldots \ldots \ldots \ldots \ldots \ldots$ & $1: 8$ & $1: 16$ & $1: 32$ & $1: 64$ & $1: 128$ & $1: 256$ & $1: 512$ & $1: 1024$ & \\
\hline & \multicolumn{9}{|l|}{ Diagnosis } & \\
\hline $\begin{array}{l}\text { L. H. } \\
\text { P. B. } \\
\text { J. W. } \\
\text { G. D. } \\
\text { C. A. J. } \\
\text { M. R. } \\
\text { W.S. } \\
\text { M. G. } \\
\text { D. G. } \\
\text { W. F. } \\
\text { A. F. } \\
\text { W. H. } \\
\text { M. B. } \\
\text { E. W. } \\
\text { A. R. } \\
\text { J. DeM. } \\
\text { M. W. } \\
\text { W. M.K. } \\
\text { S. M. } \\
\text { J.S. }\end{array}$ & $\begin{array}{l}\text { Normal } \\
\text { Normal } \\
\text { Normal } \\
\text { Normal } \\
\text { Normal } \\
\text { Carcinoma of lung } \\
\text { Retroperitoneal sarcoma } \\
\text { Pelvic inflammatory disease } \\
\text { Proteus infection } \\
\text { Proteus infection } \\
\text { Proteus infection } \\
\text { Proteus and B. Walchi infection } \\
\text { Diseeminated lupus erythematosus } \\
\text { Diseminated lupus erythematosus } \\
\text { Pneumonia, horse serum Rr. } \\
\text { Pneumonia, horse serum Rr. } \\
\text { Serum disease } \\
\text { Pneumonia, no serum } \\
\text { Hemolytic streptococcus infection } \\
\text { Hemolytic streptococcus infection }\end{array}$ & $\begin{array}{r}0 \\
++ \\
++ \\
++ \\
+ \\
++ \\
0\end{array}$ & $t_{0}$ & $\begin{array}{r}+ \\
\mathbf{0} \\
\mathbf{0} \\
\mathbf{0} \\
+ \\
\mathbf{0} \\
\mathbf{0} \\
+++ \\
+++ \\
+++ \\
+ \\
\mathbf{0} \\
\mathbf{0} \\
\mathbf{0}\end{array}$ & $\begin{array}{c}\mathbf{0} \\
\mathbf{0} \\
\mathbf{0} \\
\mathbf{0} \\
\mathbf{0} \\
+ \\
\mathbf{0} \\
\mathbf{0} \\
\mathbf{0} \\
\mathbf{0} \\
\mathbf{0} \\
\mathbf{0} \\
\mathbf{0} \\
\mathbf{0} \\
++ \\
+++\end{array}$ & $\begin{array}{l}\mathbf{0} \\
0 \\
0 \\
0 \\
0 \\
0 \\
0 \\
0 \\
0 \\
0 \\
0 \\
0 \\
0 \\
0 \\
0\end{array}$ & $\begin{array}{r}\mathbf{0} \\
\mathbf{0} \\
\mathbf{0} \\
\mathbf{0} \\
\mathbf{0} \\
\mathbf{0} \\
\mathbf{0} \\
\mathbf{0} \\
\mathbf{0} \\
\mathbf{0} \\
\mathbf{0} \\
\mathbf{0} \\
\mathbf{0} \\
\mathbf{0} \\
\mathbf{0} \\
++ \\
++ \\
+\mathbf{+}+ \\
\mathbf{0} \\
\mathbf{0} \\
\mathbf{0}\end{array}$ & $\begin{array}{l}\mathbf{0} \\
\mathbf{0} \\
\mathbf{0} \\
\mathbf{0} \\
\mathbf{0} \\
\mathbf{0} \\
\mathbf{0} \\
\mathbf{0} \\
\mathbf{0} \\
\mathbf{0} \\
\mathbf{0} \\
\mathbf{0} \\
\mathbf{0} \\
\mathbf{0} \\
\mathbf{0} \\
\mathbf{0} \\
++ \\
\mathbf{0} \\
\mathbf{0} \\
\mathbf{0}\end{array}$ & $\begin{array}{l}\mathbf{0} \\
\mathbf{0} \\
\mathbf{0} \\
\mathbf{0} \\
\mathbf{0} \\
\mathbf{0} \\
\mathbf{0} \\
\mathbf{0} \\
\mathbf{0} \\
\mathbf{0} \\
\mathbf{0} \\
\mathbf{0} \\
\mathbf{0} \\
\mathbf{0} \\
\mathbf{0} \\
\mathbf{0} \\
+ \\
\mathbf{0} \\
\mathbf{0} \\
\mathbf{0}\end{array}$ & $\begin{array}{l:l}1 & : 16 \\
1 & : 8 \\
0 & \\
1 & : 16 \\
1 & : 32 \\
1 & : 64 \\
1 & : 8 \\
1 & : 32 \\
1 & : 16 \\
1 & : 8 \\
1 & : 16 \\
1 & : 32 \\
0 & \\
0 & \\
1 & : 64 \\
1 & : 256 \\
1 & : 1024 \\
0 & \\
1 & : 16 \\
0 & \end{array}$ \\
\hline
\end{tabular}


TABLE II

Sera from cases of infectious mononucleosis. Sheep cell agglutination tests

\begin{tabular}{|c|c|c|c|c|c|c|c|c|c|c|c|c|c|}
\hline \multirow[b]{3}{*}{ Serum } & \multirow{2}{*}{\multicolumn{2}{|c|}{$\frac{\text { Serum dilution . ... }}{\text { Final dilution ...... }}$}} & $1: 2$ & $1: 4$ & $1: 8$ & $1: 16$ & $1: 32$ & $1: 64$ & 1:128 & $1: 256$ & \multirow{3}{*}{$\begin{array}{c}\text { Titer } \\
\text { (Final } \\
\text { dilution) }\end{array}$} & \multirow{3}{*}{$\begin{array}{c}\text { Wasser- } \\
\text { mann }\end{array}$} & \multirow[b]{3}{*}{ Hinton } \\
\hline & & & $1: 8$ & $1: 16$ & 1:32 & $1: 64$ & 1:128 & 1:256 & 1:512 & $1: 1024$ & & & \\
\hline & $\begin{array}{l}\text { Day } \\
\text { of } \\
\text { dis- } \\
\text { ease }\end{array}$ & Severity & & & & & & & & & & & \\
\hline $\begin{array}{l}\text { D. B. } \\
\text { D. B. } \\
\text { D. B. } \\
\text { D. B. } \\
\text { D. B. }\end{array}$ & $\begin{array}{r}4 \\
8 \\
15 \\
21 \\
37 \\
\end{array}$ & Severe & $\begin{array}{l}+++ \\
++++ \\
\neq \neq \neq \neq \\
\neq \neq \neq \neq \\
\neq \neq \neq \neq\end{array}$ & $\begin{array}{c}0 \\
++\neq+ \\
\neq \neq \neq \neq \\
\neq \neq \neq \neq \\
\neq \neq \neq \neq\end{array}$ & $\begin{array}{c}0 \\
++++ \\
++t+ \\
\neq \neq \neq \neq \\
++++\end{array}$ & $\begin{array}{c}0 \\
+++ \\
++++ \\
+++ \\
++\end{array}$ & & $\begin{array}{c}0 \\
0 \\
++ \\
++ \\
0\end{array}$ & $\begin{array}{c}0 \\
0 \\
++ \\
++\end{array}$ & $\begin{array}{l}\mathbf{0} \\
\mathbf{0} \\
+ \\
0 \\
0\end{array}$ & $\begin{array}{l}1: 8 \\
1: 128 \\
1: 1024 \\
1: 512 \\
1: 128 \\
\end{array}$ & $\begin{array}{l}\mathbf{0} \\
\mathbf{0} \\
\mathbf{0} \\
\mathbf{0} \\
\mathbf{0}\end{array}$ & $\begin{array}{l}\mathbf{0} \\
\mathbf{0} \\
\mathbf{0} \\
\mathbf{0} \\
\mathbf{0}\end{array}$ \\
\hline $\begin{array}{l}\text { A. G. } \\
\text { A. G. } \\
\text { A. G. } \\
\text { A. G. }\end{array}$ & $\begin{array}{l}15 \\
25 \\
31 \\
65\end{array}$ & Severe & $\begin{array}{l}\neq \neq \neq \neq \\
+\neq \neq \neq \\
\neq \neq \neq \neq \\
\neq \neq \neq \neq\end{array}$ & $\begin{array}{l}\neq \neq \neq \neq \\
\neq \neq \neq \neq \\
\neq+\neq \neq \\
++t+\end{array}$ & $\begin{array}{c}++++ \\
++++ \\
+++t \\
+++\end{array}$ & $\begin{array}{c}++++ \\
++++ \\
++t+ \\
+++\end{array}$ & & $\begin{array}{l}++ \\
+ \\
+\end{array}$ & $\begin{array}{l}\mathbf{0} \\
\mathbf{0} \\
\mathbf{0} \\
\mathbf{0}\end{array}$ & $\begin{array}{l}\mathbf{0} \\
\mathbf{0} \\
\mathbf{0} \\
\mathbf{0}\end{array}$ & $\begin{array}{l}1: 256 \\
1: 256 \\
1: 256 \\
1: 128\end{array}$ & $\begin{array}{l}\mathbf{0} \\
\mathbf{0} \\
\mathbf{0} \\
-\end{array}$ & $\begin{array}{l}\mathbf{0} \\
\mathbf{0} \\
\mathbf{0} \\
\mathbf{0}\end{array}$ \\
\hline $\begin{array}{l}\text { M.L.R. } \\
\text { M.L.R. } \\
\text { M. L.R. } \\
\end{array}$ & $\begin{array}{r}5 \\
17 \\
45 \\
\end{array}$ & Moderate & $\begin{array}{l}\neq \neq \neq \neq \\
\neq \neq \neq \neq \\
\neq \neq \neq \neq\end{array}$ & $\begin{array}{l}\neq \neq \neq \neq \\
\neq \neq \neq \neq \\
\neq+十 \neq\end{array}$ & $\begin{array}{l}+t+t \\
\neq \neq \neq \neq \\
t+t+\end{array}$ & $\begin{array}{l}++t+ \\
++t+t \\
++t+\end{array}$ & $\begin{array}{c}++t+ \\
++t \\
++t\end{array}$ & $\begin{array}{c}++ \\
++ \\
++\end{array}$ & $+t$ & $\stackrel{+}{+}$ & $\begin{array}{l}1: 1024 \\
1: 1024 \\
1: 512 \\
\end{array}$ & $\begin{array}{l}\mathbf{0} \\
\mathbf{0} \\
\mathbf{0}\end{array}$ & $\begin{array}{l}\mathbf{0} \\
\mathbf{0} \\
\mathbf{0} \\
\end{array}$ \\
\hline $\begin{array}{l}\text { A. H. } \\
\text { A. H. }\end{array}$ & $\begin{array}{r}8 \\
15 \\
\end{array}$ & Moderate & $\begin{array}{l}\neq \neq \neq \neq \\
\neq \neq \neq \neq\end{array}$ & $\begin{array}{l}\neq \neq \neq \neq \\
\neq \neq \neq \neq\end{array}$ & $\begin{array}{l}\neq \neq \neq \neq \\
\neq \neq \neq \neq\end{array}$ & $\begin{array}{l}+++\neq \\
\neq \neq \neq \neq\end{array}$ & $\begin{array}{l}++++ \\
\neq \neq \neq \neq\end{array}$ & $\begin{array}{c}+++ \\
++++\end{array}$ & $+\stackrel{+}{++}$ & $\stackrel{0}{++}$ & $\begin{array}{l}1: 512 \\
1: 1024+\end{array}$ & $\begin{array}{l}\mathbf{0} \\
\mathbf{0} \\
\end{array}$ & $\mathbf{0}$ \\
\hline $\begin{array}{l}\text { C. } \mathbf{M} \text {. } \\
\text { C. } \mathbf{M} \text {. }\end{array}$ & $\begin{array}{l}? 15 \\
? 23 \\
\end{array}$ & Moderate & $\begin{array}{l}\neq \neq \neq \neq \\
\neq \neq \neq \neq\end{array}$ & $\begin{array}{l}\neq \neq \neq \neq \\
\neq \neq \neq \neq\end{array}$ & $\begin{array}{l}\neq \neq \neq \neq \\
\neq \neq \neq \neq\end{array}$ & $\begin{array}{l}\neq \neq \neq \neq \\
\neq \neq \neq \neq\end{array}$ & $\begin{array}{l}\neq \neq \neq \neq \\
\neq \neq \neq \neq\end{array}$ & $\begin{array}{l}++++ \\
+++t\end{array}$ & $+t+$ & $++t$ & $\begin{array}{l}1: 1024+ \\
1: 1024+\end{array}$ & $\begin{array}{l}\mathbf{0} \\
\mathbf{0}\end{array}$ & $\begin{array}{l}\mathbf{0} \\
\mathbf{0} \\
\end{array}$ \\
\hline o.s. & $? 14$ & Mild & $\neq \neq \neq \neq$ & $\neq \neq \neq \neq$ & $\neq \neq \neq \neq$ & ++++ & ++++ & ++++ & +++ & + & 1:1024 & $\mathbf{0}$ & $\mathbf{0}$ \\
\hline R.S. & $? 21$ & Mild & ++++ & $\neq \neq \neq \neq$ & $\neq \neq \neq \neq$ & ++++ & ++++ & $++t$ & $++t$ & + & 1:1024 & $\mathbf{0}$ & $\mathbf{0}$ \\
\hline $\begin{array}{l}\text { A. L. } \\
\text { A. L. }\end{array}$ & $\begin{array}{l}10 \\
15 \\
\end{array}$ & Questionable & $\begin{array}{l}++++ \\
++++\end{array}$ & $\begin{array}{l}++++ \\
++++\end{array}$ & $\begin{array}{l}+++ \\
++++\end{array}$ & $+t$ & $\begin{array}{c}0 \\
++\end{array}$ & $\begin{array}{l}\mathbf{0} \\
\mathbf{0}\end{array}$ & $\begin{array}{l}\mathbf{0} \\
\mathbf{0}\end{array}$ & $\begin{array}{l}\mathbf{0} \\
\mathbf{0}\end{array}$ & $\begin{array}{l}1: 64 \\
1: 128\end{array}$ & $\begin{array}{l}\mathbf{0} \\
\mathbf{0}\end{array}$ & $\begin{array}{l}\mathbf{0} \\
\mathbf{0}\end{array}$ \\
\hline P. M. & $? 15$ & Mild & $\neq \neq \neq \neq$ & $\neq \neq \neq \neq$ & $\neq \neq \neq \neq$ & $+++t$ & ++++ & +++ & $+t$ & ++ & 1:1024 & 0 & $\mathbf{0}$ \\
\hline R. B. & 9 & Questionable & $+t$ & $\mathbf{0}$ & $\mathbf{0}$ & $\mathbf{0}$ & 0 & o & o & $\mathbf{0}$ & $1: 8$ & 0 & $\mathbf{0}$ \\
\hline D. $\mathbf{M}$. & 10 & Mild & $\neq \neq \neq \neq$ & $\neq \neq \neq \neq$ & $\neq \neq \neq \neq$ & $\neq \neq \neq \neq$ & ++++ & ++++ & ++++ & +++ & 1:1024+ & $\mathbf{0}$ & $\mathbf{0}$ \\
\hline A. $\mathbf{G}$. & $?$ & Questionable & $+++t$ & ++++ & $+++t$ & $++t+$ & ++++ & $+++t$ & $+t$ & 0 & $1: 512$ & 0 & 0 \\
\hline $\begin{array}{l}\text { M. L. } \\
\text { M. L. }\end{array}$ & $\begin{array}{l}24 \\
82\end{array}$ & Very severe & $+\overline{+} \overline{+}+$ & ++++ & $\begin{array}{c}++++ \\
+++\end{array}$ & $\begin{array}{c}++ \\
++\end{array}$ & ++ & $t_{0}^{+}$ & $\stackrel{+}{0}$ & $\begin{array}{l}\mathbf{0} \\
\mathbf{0}\end{array}$ & $\begin{array}{l}1: 512 \\
1: 128\end{array}$ & o & $\underline{0}$ \\
\hline
\end{tabular}

* Represents very strong reaction.

noted that most of these fall within the normal range given by most authors, namely $1: 64$ (final dilution) or less. The three exceptions occur in the sera of patients who had received horse serum for the treatment of pneumonia, and the highest titer, $1: 1024$, was recorded in a patient with serum disease. It has been known for some time that the injection of horse serum would lead to the production of "heterophile" antibodies. Davidsohn (13) has shown that these may be distinguished from the sheep cell agglutinins of infectious mononucleosis by their disappearance following absorption of the serum with fresh guinea pig kidney.

In Table II are recorded the results of sheep cell agglutination, Wassermann, and Hinton tests on 13 cases of clinically diagnosed infectious mononucleosis. In this group no false positive serological reactions for syphilis were noted, al- though the last patient, M. L., ${ }^{3}$ had a positive spinal fluid Hinton when the blood Hinton was negative. All but 1 case, R. B., in whom the diagnosis was questionable, showed a marked elevation of the titer of sheep cell agglutinins. D. B., our most adequately studied case, was followed from the fourth day until complete recovery occurred. In his serum the titer was observed to rise from a normal level of $1: 8$ on the fourth day to a peak of $1: 1024$ on the fifteenth day, and then gradually to fall back toward normal. These 13 cases represent a fair cross section of the disease, with the severity varying markedly. In accordance with the observations of others, there was no correlation between the severity of the disease and the titer of sheep cell agglutinins attained.

3 The authors wish to express their appreciation to Dr. Ruth B. Thomas, Dover-Foxcroft, Maine, for sending the serum samples from this patient. 
TABLE III

Control sera. Listerella agglutination

\begin{tabular}{|c|c|c|c|c|c|c|c|c|}
\hline Serum dilution. .......... & $1: 10$ & $1: 20$ & $1: 40$ & $1: 80$ & $1: 160$ & $1: 320$ & \multirow{2}{*}{\multicolumn{2}{|c|}{$\begin{array}{c}\text { Titer } \\
\text { (Final dilution) }\end{array}$}} \\
\hline Final dilution........... & $1: 20$ & $1: 40$ & $1: 80$ & $1: 160$ & $1: 320$ & $1: 640$ & & \\
\hline Serum & & & & & & & vs. C.H. & vs. UM. \\
\hline L. H........... & $\begin{array}{l}0^{*} \\
0 \dagger\end{array}$ & $\begin{array}{l}\mathbf{0} \\
\mathbf{0}\end{array}$ & $\begin{array}{l}\mathbf{0} \\
\mathbf{0}\end{array}$ & $\begin{array}{l}\mathbf{0} \\
\mathbf{0}\end{array}$ & $\begin{array}{l}\mathbf{0} \\
\mathbf{0}\end{array}$ & $\begin{array}{l}\mathbf{0} \\
\mathbf{0}\end{array}$ & 0 & 0 \\
\hline P. B......... & $\stackrel{+}{++}$ & $\begin{array}{c}0 \\
++\end{array}$ & $\begin{array}{l}\mathbf{0} \\
\mathbf{0}\end{array}$ & $\begin{array}{l}\mathbf{0} \\
\mathbf{0}\end{array}$ & $\begin{array}{l}\mathbf{0} \\
\mathbf{0}\end{array}$ & $\begin{array}{l}0 \\
0\end{array}$ & $1: 20$ & $1: 40$ \\
\hline $\mathbf{J} . \mathbf{W} \ldots \ldots \ldots \ldots \ldots$ & $\begin{array}{l} \pm \\
t\end{array}$ & $\begin{array}{l}0 \\
0\end{array}$ & $\begin{array}{l}0 \\
0\end{array}$ & $\begin{array}{l}\mathbf{0} \\
\mathbf{0}\end{array}$ & $\begin{array}{l}\mathbf{0} \\
\mathbf{0}\end{array}$ & $\begin{array}{l}0 \\
0\end{array}$ & 0 & $1: 20$ \\
\hline G. D....... & $+\underset{0}{+}+$ & $+t$ & $\begin{array}{l}0 \\
0\end{array}$ & $\begin{array}{l}\mathbf{0} \\
\mathbf{0}\end{array}$ & $\begin{array}{l}\mathbf{0} \\
\mathbf{0}\end{array}$ & $\begin{array}{l}\mathbf{0} \\
\mathbf{0}\end{array}$ & $1: 40$ & 0 \\
\hline C. J........... & $\begin{array}{c}0 \\
++\end{array}$ & $\begin{array}{l}0 \\
\pm\end{array}$ & $\begin{array}{l}0 \\
0\end{array}$ & $\begin{array}{l}\mathbf{0} \\
\mathbf{0}\end{array}$ & $\begin{array}{l}\mathbf{0} \\
\mathbf{0}\end{array}$ & $\begin{array}{l}\mathbf{0} \\
\mathbf{0}\end{array}$ & $\mathbf{0}$ & $1: 20$ \\
\hline M. R........... & $\begin{array}{c}+++ \\
+\end{array}$ & $+\underset{0}{+}+$ & $\stackrel{+}{0}$ & $\begin{array}{l}\mathbf{0} \\
\mathbf{0}\end{array}$ & $\begin{array}{l}0 \\
0\end{array}$ & $\begin{array}{l}0 \\
0\end{array}$ & $1: 80$ & $1: 20$ \\
\hline W. S......... & $\begin{array}{l}+++t \\
+++t\end{array}$ & $\begin{array}{l}+t \\
++\end{array}$ & $\begin{array}{l}0 \\
0\end{array}$ & $\begin{array}{l}\mathbf{0} \\
\mathbf{0}\end{array}$ & $\begin{array}{l}0 \\
0\end{array}$ & $\begin{array}{l}0 \\
0\end{array}$ & $1: 40$ & $1: 40$ \\
\hline M. G........... & + & $\begin{array}{l}\mathbf{0} \\
\mathbf{0}\end{array}$ & $\begin{array}{l}\mathbf{0} \\
\mathbf{0}\end{array}$ & $\begin{array}{l}\mathbf{0} \\
\mathbf{0}\end{array}$ & $\begin{array}{l}\mathbf{0} \\
\mathbf{0}\end{array}$ & $\begin{array}{l}\mathbf{0} \\
\mathbf{0}\end{array}$ & $1: 20$ & $1: 20$ \\
\hline D. G.............. & $\begin{array}{l} \pm \\
+\end{array}$ & $\begin{array}{l}0 \\
0\end{array}$ & $\begin{array}{l}0 \\
0\end{array}$ & $\begin{array}{l}\mathbf{0} \\
0\end{array}$ & $\begin{array}{l}\mathbf{0} \\
0\end{array}$ & $\begin{array}{l}0 \\
0\end{array}$ & $\mathbf{0}$ & $1: 20$ \\
\hline W. F............. & $\begin{array}{c}0 \\
++\end{array}$ & $\begin{array}{l}\mathbf{0} \\
\mathbf{0}\end{array}$ & $\begin{array}{l}\mathbf{0} \\
\mathbf{0}\end{array}$ & $\begin{array}{l}\mathbf{0} \\
\mathbf{0}\end{array}$ & $\begin{array}{l}0 \\
0\end{array}$ & $\begin{array}{l}0 \\
0\end{array}$ & $\mathbf{0}$ & $1: 20$ \\
\hline A. F........ & $\begin{array}{l}0 \\
0\end{array}$ & $\begin{array}{l}\mathbf{0} \\
\mathbf{0}\end{array}$ & $\begin{array}{l}0 \\
0\end{array}$ & $\begin{array}{l}\mathbf{0} \\
\mathbf{0}\end{array}$ & $\begin{array}{r}\mathbf{0} \\
.0\end{array}$ & $\begin{array}{l}0 \\
0\end{array}$ & 0 & 0 \\
\hline W. H........... & $\begin{array}{l}0 \\
0\end{array}$ & $\begin{array}{l}\mathbf{0} \\
\mathbf{0}\end{array}$ & $\begin{array}{l}0 \\
0\end{array}$ & $\begin{array}{l}\mathbf{0} \\
\mathbf{0}\end{array}$ & $\begin{array}{l}0 \\
0\end{array}$ & $\begin{array}{l}0 \\
0\end{array}$ & $\mathbf{0}$ & 0 \\
\hline M. B......... & $\stackrel{ \pm}{+}+$ & $\begin{array}{l}0 \\
+\end{array}$ & $\begin{array}{l}0 \\
0\end{array}$ & $\begin{array}{l}\mathbf{0} \\
\mathbf{0}\end{array}$ & $\begin{array}{l}\mathbf{0} \\
\mathbf{0}\end{array}$ & $\begin{array}{l}0 \\
0\end{array}$ & $\mathbf{0}$ & $1: 40$ \\
\hline E. W............. & $\stackrel{+}{++t}$ & $\begin{array}{c}0 \\
++\end{array}$ & $\begin{array}{l}0 \\
\mathbf{0}\end{array}$ & $\begin{array}{l}\mathbf{0} \\
\mathbf{0}\end{array}$ & $\begin{array}{l}0 \\
0\end{array}$ & $\begin{array}{l}0 \\
0\end{array}$ & $1: 20$ & $1: 40$ \\
\hline A. R.......... & $\begin{array}{c}++ \\
+\end{array}$ & $\begin{array}{l}\mathbf{0} \\
\mathbf{0}\end{array}$ & $\begin{array}{l}0 \\
0\end{array}$ & $\begin{array}{l}\mathbf{0} \\
\mathbf{0}\end{array}$ & $\begin{array}{l}\mathbf{0} \\
\mathbf{0}\end{array}$ & $\begin{array}{l}0 \\
0\end{array}$ & $1: 20$ & $1: 20$ \\
\hline J. DeM....... & + & + & $\begin{array}{l}\mathbf{0} \\
\mathbf{0}\end{array}$ & $\begin{array}{l}\mathbf{0} \\
\mathbf{0}\end{array}$ & $\begin{array}{l}\mathbf{0} \\
\mathbf{0}\end{array}$ & $\begin{array}{l}0 \\
0\end{array}$ & $1: 40$ & $1: 40$ \\
\hline M. W.......... & $\stackrel{0}{++}$ & $\begin{array}{l}0 \\
\pm\end{array}$ & $\begin{array}{l}0 \\
0\end{array}$ & $\begin{array}{l}\mathbf{0} \\
\mathbf{0}\end{array}$ & $\begin{array}{l}\mathbf{0} \\
\mathbf{0}\end{array}$ & $\begin{array}{l}\mathbf{0} \\
\mathbf{0}\end{array}$ & 0 & $1: 20$ \\
\hline W. Mck. . . . . . . . & $+\stackrel{ \pm}{+}+$ & $\begin{array}{l}0 \\
\pm\end{array}$ & $\begin{array}{l}\mathbf{0} \\
\mathbf{0}\end{array}$ & $\begin{array}{l}\mathbf{0} \\
\mathbf{0}\end{array}$ & $\begin{array}{l}\mathbf{0} \\
\mathbf{0}\end{array}$ & $\begin{array}{l}\mathbf{0} \\
\mathbf{0}\end{array}$ & 0 & $1: 20$ \\
\hline S. $\mathbf{M} \ldots \ldots \ldots \ldots \ldots$ & $+\underset{0}{+}+$ & $\begin{array}{l}\mathbf{0} \\
\mathbf{0}\end{array}$ & $\begin{array}{l}\mathbf{0} \\
\mathbf{0}\end{array}$ & $\begin{array}{l}\mathbf{0} \\
\mathbf{0}\end{array}$ & $\begin{array}{l}\mathbf{0} \\
\mathbf{0}\end{array}$ & $\begin{array}{l}\mathbf{0} \\
\mathbf{0}\end{array}$ & $1: 20$ & $\mathbf{0}$ \\
\hline J. S........ & $\stackrel{ \pm}{++}$ & $\begin{array}{l}\mathbf{0} \\
\mathbf{0}\end{array}$ & $\begin{array}{l}\mathbf{0} \\
\mathbf{0}\end{array}$ & $\begin{array}{l}\mathbf{0} \\
\mathbf{0}\end{array}$ & $\begin{array}{l}\mathbf{0} \\
\mathbf{0}\end{array}$ & $\begin{array}{l}\mathbf{0} \\
\mathbf{0}\end{array}$ & 0 & $1: 20$ \\
\hline $\begin{array}{c}\text { Pooled immune rabbit } \\
\text { serum.............. }\end{array}$ & $\begin{array}{l}++t+ \\
++t+\end{array}$ & $\begin{array}{l}+\neq+t \\
\neq \neq \neq \neq\end{array}$ & $t+t$ & $++t-1$ & $\begin{array}{l}+t+ \\
++t+\end{array}$ & $\begin{array}{c}+t+ \\
+t+t\end{array}$ & $1: 640$ & $1: 640+$ \\
\hline
\end{tabular}


Tables III and IV record the results of agglutination tests with representative strains of the two serological groups of the genus Listerella. The data require little comment. The recorded titers are slightly higher for the cases of infectious mononucleosis than for the controls. No definite trends are observed in any particular case, the variations between samples being within the limits of error, with the possible exception of the last case, M. L. However, in her serum the titer for $C$. $H$. rose slightly, while that for $U M$. fell during recovery.

\section{DISCUSSION}

This study was undertaken in order to see whether patients with infectious mononucleosis develop agglutinins for either of the two known serological groups of the genus Listerella. Although we have observed slightly higher agglutinin titers in the sera of infectious mononucleosis patients than in the sera of normal people, or of those suffering from other diseases, the interpretation of this finding is difficult. The numbers in either series are not sufficiently large to eliminate the possibility that chance variation may account for the observed differences. It might be an example of the anamnestic reaction, but many of the control sera were taken from patients with acute illnesses, and the sera of infectious mononucleosis patients failed to show a reduction in titer when they were obtained during convalescence.

In view of Kolmer's (8) results, which we have confirmed, (9) the increased agglutination of Listerella cannot be due to the presence of

TABLE IV

Sera from cases of infectious mononucleosis. Listerella agglutination

\begin{tabular}{|c|c|c|c|c|c|c|c|c|c|c|}
\hline \multirow{3}{*}{ Serum } & \multirow{2}{*}{\multicolumn{2}{|c|}{$\begin{array}{l}\text { Serum dilution } \\
\text { Final dilution } \\
\end{array}$}} & 1:10 & $1: 20$ & $1: 40$ & $1: 80$ & 1:160 & 1:320 & \multirow{2}{*}{\multicolumn{2}{|c|}{$\begin{array}{c}\text { Titer } \\
\text { (Final dilution) }\end{array}$}} \\
\hline & & & $1: 20$ & $1: 40$ & $1: 80$ & 1:160 & $1: 320$ & $1: 640$ & & \\
\hline & $\begin{array}{l}\text { Day of } \\
\text { disease }\end{array}$ & Severity & & & & & & & Vs. C.H. & Vs. UM. \\
\hline \multirow[t]{5}{*}{ D. B. } & 4 & Severe & $\begin{array}{c}++* \\
+++t\end{array}$ & $\begin{array}{c}0 \\
++\end{array}$ & $\begin{array}{l}\mathbf{0} \\
\mathbf{0}\end{array}$ & $\begin{array}{l}\mathbf{0} \\
\mathbf{0}\end{array}$ & $\begin{array}{l}\mathbf{0} \\
\mathbf{0}\end{array}$ & $\begin{array}{l}\mathbf{0} \\
\mathbf{0}\end{array}$ & $1: 20$ & $1: 40$ \\
\hline & 8 & & $+\stackrel{+}{+}+$ & $\stackrel{0}{++}$ & $\begin{array}{l}\mathbf{0} \\
\mathbf{0}\end{array}$ & $\begin{array}{l}0 \\
0\end{array}$ & $\begin{array}{l}\mathbf{0} \\
\mathbf{0}\end{array}$ & $\begin{array}{l}\mathbf{0} \\
\mathbf{0}\end{array}$ & $1: 20$ & $1: 40$ \\
\hline & 15 & & $+\stackrel{+}{+}$ & $\stackrel{0}{++}$ & $\begin{array}{l}0 \\
\pm\end{array}$ & $\begin{array}{l}\mathbf{0} \\
\mathbf{0}\end{array}$ & $\begin{array}{l}\mathbf{0} \\
\mathbf{0}\end{array}$ & $\begin{array}{l}\mathbf{0} \\
\mathbf{0}\end{array}$ & $1: 20$ & $1: 40$ \\
\hline & 21 & & $+\stackrel{ \pm}{+}+$ & $\begin{array}{l}\mathbf{0} \\
+\end{array}$ & $\begin{array}{l}\mathbf{0} \\
\mathbf{0}\end{array}$ & $\begin{array}{l}\mathbf{0} \\
\mathbf{0}\end{array}$ & $\begin{array}{l}\mathbf{0} \\
\mathbf{0}\end{array}$ & $\begin{array}{l}\mathbf{0} \\
\mathbf{0}\end{array}$ & 0 & $1: 40$ \\
\hline & 37 & & $+\stackrel{+}{+}$ & $\stackrel{0}{++}$ & $\begin{array}{l}\mathbf{0} \\
\mathbf{0} \\
\end{array}$ & $\begin{array}{l}\mathbf{0} \\
\mathbf{0} \\
\end{array}$ & $\begin{array}{l}\mathbf{0} \\
\mathbf{0} \\
\end{array}$ & $\begin{array}{l}\mathbf{0} \\
\mathbf{0} \\
\end{array}$ & $1: 20$ & $1: 40$ \\
\hline \multirow[t]{4}{*}{ A. $G$. } & 15 & Severe & + & $\begin{array}{l}0 \\
0\end{array}$ & $\begin{array}{l}0 \\
0\end{array}$ & $\begin{array}{l}0 \\
0\end{array}$ & $\begin{array}{l}0 \\
0\end{array}$ & $\begin{array}{l}\mathbf{0} \\
\mathbf{0}\end{array}$ & $1: 20$ & $1: 40$ \\
\hline & 25 & & $\stackrel{+}{+}$ & $\begin{array}{l}\mathbf{0} \\
\mathbf{0}\end{array}$ & $\begin{array}{l}\mathbf{0} \\
\mathbf{0}\end{array}$ & $\begin{array}{l}\mathbf{0} \\
\mathbf{0}\end{array}$ & $\begin{array}{l}0 \\
0\end{array}$ & $\begin{array}{l}\mathbf{0} \\
\mathbf{0}\end{array}$ & 0 & $1: 40$ \\
\hline & 31 & & $\stackrel{+}{++}$ & $\begin{array}{l}0 \\
\pm\end{array}$ & $\begin{array}{l}\mathbf{0} \\
\mathbf{0}\end{array}$ & $\begin{array}{l}\mathbf{0} \\
\mathbf{0}\end{array}$ & $\begin{array}{l}\mathbf{0} \\
\mathbf{0}\end{array}$ & $\begin{array}{l}\mathbf{0} \\
\mathbf{0}\end{array}$ & $1: 20$ & $1: 40$ \\
\hline & 65 & & $\stackrel{ \pm}{++}$ & $\begin{array}{l}\mathbf{0} \\
\mathbf{0}\end{array}$ & $\begin{array}{l}\mathbf{0} \\
\mathbf{0}\end{array}$ & $\begin{array}{l}0 \\
0\end{array}$ & $\begin{array}{l}\mathbf{0} \\
\mathbf{0}\end{array}$ & $\begin{array}{l}\mathbf{0} \\
\mathbf{0} \\
\end{array}$ & 0 & $1: 40$ \\
\hline \multirow[t]{3}{*}{ M. L. R. } & 5 & Moderate & + & $\begin{array}{l}0 \\
+\end{array}$ & $\begin{array}{l}\mathbf{0} \\
\mathbf{0}\end{array}$ & $\begin{array}{l}\mathbf{0} \\
\mathbf{0}\end{array}$ & $\begin{array}{l}\mathbf{0} \\
\mathbf{0}\end{array}$ & $\begin{array}{l}\mathbf{0} \\
\mathbf{0}\end{array}$ & $1: 20$ & $1: 40$ \\
\hline & 17 & & $+\stackrel{+}{+}$ & $\begin{array}{l}0 \\
+\end{array}$ & $\begin{array}{l}\mathbf{0} \\
\mathbf{0}\end{array}$ & $\begin{array}{l}\mathbf{0} \\
\mathbf{0}\end{array}$ & $\begin{array}{l}\mathbf{0} \\
\mathbf{0}\end{array}$ & $\begin{array}{l}\mathbf{0} \\
\mathbf{0}\end{array}$ & $1: 20$ & $1: 40$ \\
\hline & 45 & & $\stackrel{ \pm}{+t}$ & $\begin{array}{l}0 \\
\pm\end{array}$ & $\begin{array}{l}\mathbf{0} \\
\mathbf{0}\end{array}$ & $\begin{array}{l}\mathbf{0} \\
\mathbf{0}\end{array}$ & $\begin{array}{l}\mathbf{0} \\
\mathbf{0}\end{array}$ & $\begin{array}{l}\mathbf{0} \\
\mathbf{0}\end{array}$ & 0 & $1: 20$ \\
\hline
\end{tabular}


TABLE IV-Continued

\begin{tabular}{|c|c|c|c|c|c|c|c|c|c|c|}
\hline \multirow{3}{*}{ Serum } & \multirow{2}{*}{\multicolumn{2}{|c|}{$\begin{array}{l}\text { Serum dilution } \\
\text { Final dilution }\end{array}$}} & 1:10 & 1:20 & $1: 40$ & $1: 80$ & $1: 160$ & 1:320 & \multirow{2}{*}{\multicolumn{2}{|c|}{$\begin{array}{c}\text { Titer } \\
\text { (Final dilution) }\end{array}$}} \\
\hline & & & $1: 20$ & $1: 40$ & \multirow[t]{2}{*}{$1: 80$} & \multirow[t]{2}{*}{$1: 160$} & \multirow[t]{2}{*}{$1: 320$} & \multirow[t]{2}{*}{$1: 640$} & & \\
\hline & \begin{tabular}{|l|}
$\begin{array}{l}\text { Day of } \\
\text { disease }\end{array}$ \\
\end{tabular} & Severity & & & & & & & Vs. C.H. & Vs. UM. \\
\hline \multirow[t]{2}{*}{ A. $\mathrm{H}$. } & 8 & Moderate & $\stackrel{+}{++}$ & + & $\begin{array}{l}0 \\
+\end{array}$ & $\begin{array}{l}0 \\
0\end{array}$ & $\begin{array}{l}\mathbf{0} \\
\mathbf{0}\end{array}$ & $\begin{array}{l}\mathbf{0} \\
\mathbf{0}\end{array}$ & $1: 20$ & $1: 80$ \\
\hline & 15 & & $\begin{array}{c}+++ \\
++++\end{array}$ & $\stackrel{+}{++}$ & $\begin{array}{c}0 \\
++\end{array}$ & $\begin{array}{l}0 \\
0\end{array}$ & $\begin{array}{l}0 \\
0\end{array}$ & $\begin{array}{l}0 \\
0\end{array}$ & 1:40 & $1: 80$ \\
\hline \multirow[t]{2}{*}{ C. M. } & $? 15$ & Moderate & $\begin{array}{l}+++ \\
++\end{array}$ & + & $\begin{array}{l}\mathbf{0} \\
\mathbf{0}\end{array}$ & $\begin{array}{l}0 \\
0\end{array}$ & $\begin{array}{l}\mathbf{0} \\
\mathbf{0}\end{array}$ & $\begin{array}{l}\mathbf{0} \\
\mathbf{0}\end{array}$ & 1:40 & $1: 40$ \\
\hline & $? 23$ & & $\begin{array}{l}++ \\
++\end{array}$ & $\begin{array}{l}0 \\
\pm\end{array}$ & $\begin{array}{l}\mathbf{0} \\
\mathbf{0}\end{array}$ & $\begin{array}{l}\mathbf{0} \\
\mathbf{0}\end{array}$ & $\begin{array}{l}0 \\
0\end{array}$ & $\begin{array}{l}0 \\
0\end{array}$ & $1: 20$ & $1: 20$ \\
\hline o.s. & $? 14$ & Mild & $\overline{t+-}$ & $\bar{t} \overline{+}$ & $-\frac{-}{+}$ & $-\frac{-}{0}-$ & $-\frac{-}{0}-$ & $-\frac{-}{0}-$ & --- & 1:80 \\
\hline R. S. & $? 21$ & Mild & $\begin{array}{l}++t \\
++t\end{array}$ & $\begin{array}{l} \pm \\
\pm\end{array}$ & $\begin{array}{l}\mathbf{0} \\
\mathbf{0}\end{array}$ & $\begin{array}{l}0 \\
0\end{array}$ & $\begin{array}{l}\mathbf{0} \\
\mathbf{0}\end{array}$ & $\begin{array}{l}\mathbf{0} \\
\mathbf{0}\end{array}$ & $1: 20$ & $1: 20$ \\
\hline \multirow[t]{2}{*}{ A. $\mathrm{L}$. } & 10 & Questionable & + & $\stackrel{+}{0}$ & $\begin{array}{l}\mathbf{0} \\
\mathbf{0}\end{array}$ & $\begin{array}{l}\mathbf{0} \\
\mathbf{0}\end{array}$ & $\begin{array}{l}\mathbf{0} \\
\mathbf{0}\end{array}$ & $\begin{array}{l}\mathbf{0} \\
\mathbf{0}\end{array}$ & 1:40 & $1: 20$ \\
\hline & 15 & & + & $\begin{array}{l}0 \\
+\end{array}$ & $\begin{array}{l}\mathbf{0} \\
\mathbf{0}\end{array}$ & $\begin{array}{l}0 \\
0\end{array}$ & $\begin{array}{l}0 \\
0\end{array}$ & $\begin{array}{l}\mathbf{0} \\
\mathbf{0}\end{array}$ & $1: 20$ & $1: 40$ \\
\hline P. M. & $? 15$ & Mild & $\begin{array}{l}+++ \\
+++\end{array}$ & $+\stackrel{+}{+}+$ & $\begin{array}{l}0 \\
+\end{array}$ & $\begin{array}{l}\mathbf{0} \\
\mathbf{0}\end{array}$ & $\begin{array}{l}\mathbf{0} \\
\mathbf{0}\end{array}$ & $\begin{array}{l}\mathbf{0} \\
\mathbf{0}\end{array}$ & 1:40 & $1: 80$ \\
\hline R. B. & 9 & Questionable & $\stackrel{+}{++}$ & $\begin{array}{l}0 \\
\pm\end{array}$ & $\begin{array}{l}0 \\
0\end{array}$ & $\begin{array}{l}0 \\
0\end{array}$ & $\begin{array}{l}0 \\
0\end{array}$ & $\begin{array}{l}0 \\
0\end{array}$ & $1: 20$ & $1: 20$ \\
\hline D. M. & 10 & Mild & $\begin{array}{c}+++ \\
++++\end{array}$ & $\begin{array}{c}++ \\
++t\end{array}$ & $\begin{array}{c}0 \\
++\end{array}$ & $\begin{array}{l}0 \\
0\end{array}$ & $\begin{array}{l}0 \\
0\end{array}$ & $\begin{array}{l}\mathbf{0} \\
\mathbf{0}\end{array}$ & $1: 40$ & $1: 80$ \\
\hline A. G. & $?$ & Questionable & $\begin{array}{l}+++t \\
+++\end{array}$ & $\begin{array}{c}+++ \\
++\end{array}$ & + & $\begin{array}{l}0 \\
0\end{array}$ & $\begin{array}{l}\mathbf{0} \\
\mathbf{0}\end{array}$ & $\begin{array}{l}0 \\
0\end{array}$ & $1: 80$ & $1: 80$ \\
\hline \multirow[t]{2}{*}{ M. L. } & 24 & Very severe & $\begin{array}{l}--- \\
---\end{array}$ & $\begin{array}{c}+++ \\
++++\end{array}$ & $\stackrel{++}{++}+$ & $\stackrel{+}{++}$ & $\stackrel{ \pm}{+}$ & $\begin{array}{l}0 \\
0\end{array}$ & $1: 160$ & $1: 320$ \\
\hline & 82 & & $\begin{array}{l}-ー-ー \\
----\end{array}$ & $\begin{array}{l}++++ \\
+++\end{array}$ & $\begin{array}{c}+++ \\
++\end{array}$ & $\begin{array}{c}++ \\
+\end{array}$ & \pm & $\begin{array}{l}\mathbf{0} \\
\mathbf{0}\end{array}$ & $1: 160$ & $1: 160$ \\
\hline
\end{tabular}

sheep cell agglutinin, since anti-Listerella sera do not agglutinate sheep cells, nor do high titer antisheep cell sera agglutinate Listerella.

Perhaps the strongest point against the significance of the increased titer found in the mononucleosis patients is the lack of any definite trend either upward or downward during the progression of the disease and recovery from it.

Unfortunately, we did not have access to $\mathrm{Ny}$ feldt's organisms obtained from what seem to be proven cases of infectious mononucleosis, so that we do not know whether they represent a different serological group. If this were the case, a distant antigenic relationship might explain the slight increase in titer found with our strains. However, we received Julianelle's 4 type strains, one of which was obtained from the blood of a mononucleosis patient, and they fall into the two known groups so that this seems unlikely. One is forced to conclude that, if infectious mononucleosis is caused by a member of the genus Listerella, the organisms remain localized, producing an early slight rise in agglutinin titer, and persist for long periods of time, thus maintaining this titer long into convalescence. The latter is not impossible, in view of the prolonged, insidious course of the disease in many patients. However, it is evident

4 The authors wish to express their appreciation to Dr. L. A. Julianelle for sending these strains and for making his results available to us before their publication. 
that unless the interpretation of our data is considerably strained, there is no conclusive serological evidence for an etiological relationship between the known strains of Listerella and infectious mononucleosis.

Since the completion of this work, a report by Julianelle (14) has appeared which, in a larger series of cases, records results similar to ours. He failed to grow Listerella from the blood in 17 out of 18 patients with infectious mononucleosis, the one exception being the case previously reported. We have studied throat cultures from 6 patients, blood cultures from 5 acutely ill patients, and a lymph node from a patient in the florid phase of the disease with a sheep cell agglutination titer of $1: 512$, and have never isolated any organisms resembling Listerella. Julianelle also reported finding a slightly elevated Listerella agglutination titer in 13 out of 35 patients with mononucleosis.

\section{SUM MARY}

1. The sera of 5 normal adults, 15 patients with miscellaneous diseases, and 13 patients with infectious mononucleosis, were tested for agglutinins against sheep cells and a representative strain of each of the two known serological groups of the genus Listerella (Bact. monocytogenes).

2. The results of the sheep cell agglutination tests were in accordance with the findings of many previous workers.

3. The results of the Listerella agglutination tests showed a slightly elevated titer in the sera of the patients with infectious mononucleosis as compared to the control sera.

4. There was no significant trend upward or downward in the Listerella agglutinin titer during the course of the disease or recovery from it.

5. It is concluded that this study does not suggest any definite etiological relationship between the known Listerella organisms and infectious mononucleosis.

\section{BIBLIOGRAPHY}

1. Murray, E. G. D., Webb, R. A., and Swann, M. B. R., Disease of rabbits characterized by large mononuclear leucocytosis, caused by hitherto undescribed bacillus, Bacterium monocytogenes. J. Path. and Bact., 1926, 29, 407.

2. Seastone, C. V., Pathogenic organisms of the genus Listerella. J. Exper. Med., 1935, 62, 203.

3. Nyfeldt, A., Klinische und experimentelle Untersuchungen ueber die mononucleosis infectiosa. Folia haemat., 1932, 47, 1.

4. Schmidt, V., and Nyfeldt, A., Infectious mononucleosis and meningoencephalitis. Ugesk. f. laeger., 1938, 100, 336. Abst. J. A. M. A., 1938, 110, 1884.

5. Pons, C. A., and Julianelle, L. A., Isolation of Listerella monocytogenes from infectious mononucleosis. Proc. Soc. Exper. Biol. and Med., 1939, 40, 360.

6. Baldridge, C. W., Rohner, F. J., and Hansmann, G. H., Glandular fever (infectious mononucleosis). Arch. Int. Med., 1926, 38, 413.

7. Wising, P. J., Some experiments with lymph gland material from cases of infectious mononucleosis. Acta. med. Scandinav., 1939, 98, 328.

8. Kolmer, J. A., Listerella monocytogenes in relation to the Wassermann and flocculation reactions in normal rabbits. Proc. Soc. Exper. Biol. and Med., 1939, 42, 183.

9. Dammin, G. J., Bradley, M. A., and Janeway, C. A., Studies on infectious mononucleosis I. The Listerella organisms : bacteriology, immunological relationships, and infection in experimental animals. (In preparation.)

10. Carey, B. W., Infection with an organism of the genus Listerella. J. Pediat., 1936, 8, 626.

11. Julianelle, L. A., and Pons, C. A., Immunological and serological reactions of Listerella monocytogenes. Proc. Soc. Exper. Biol. and Med., 1939, 40, 364.

12. Paul, J. R., and Bunnell, W. W., The presence of heterophile antibodies in infectious mononucleosis. Am. J. Med. Sc., 1932, 183, 90.

13. Davidsohn, I., Test for infectious mononucleosis. Am. J. Clin. Path., Tech. Supp., 1938, 2, 56.

14. Julianelle, L. A., The function of Listerella in infection. Ann. Int. Med., 1940, 14, 608. 